\title{
Dithiocarbamate promoted practical synthesis of $N$-Aryl-2-aminobenzazoles: Synthesis of novel Aurora-A kinase inhibitor
}

\author{
NARESH KUMAR KATARI*, M VENKATANARAYANA and KUMMARI SRINIVAS \\ Department of Chemistry, HTP Campus, GITAM University, Rudraram, Hyderabad, Telangana 502 329, India \\ e-mail: dr.n.k.katari@gmail.com
}

MS received 18 June 2014; revised 24 July 2014; accepted 10 August 2014

\begin{abstract}
Various $N$-aryl-2-aminobenzoxazoles and $N$-aryl-2-aminobenzothiazoles were synthesized from $o$-aminophenol and $o$-aminothiophenol, respectively, mediated by dithiocarbamate in one step. The salient features of this method include mild reaction condition, high yield and large scale synthesis. Application of this methodology has been demonstrated by synthesizing potent Aurora kinase-A inhibitors.
\end{abstract}

Keywords. 2-Aminobenzoxazole; 2-Aminobenzothiazole; Dithiocarbamate; 4-Aminoquinazoline Aurora kinase.

\section{Introduction}

Benzo-1, 3-diazoles are a biologically important class of molecules and are widely used as pharmaceutical agents. Interestingly, during the structure activity relationship (SAR) studies it was observed that the change of the structure of substituent group at C2 position commonly results in change of its bioactivity. 2-substituted benzoxazoles, particularly $N$-aryl2-aminobenzoxazole derivatives, have been or are currently under investigation for the treatment of a wide variety of disorders such as HIV, ${ }^{1}$ neuro degeneration $^{2}$ and inflammatory diseases. ${ }^{3}$ Moreover, 2-aminobenzoxazoles have also been reported as VEGFR inhibitors and are thus important for hyper proliferative diseases such as cancer and rheumatoid arthritis. ${ }^{4}$ The 2 -aminobenzothiazole compounds were mostly synthesized as kinase inhibitors in recent times. For example, the investigations of 2-amino benzothiazole as a key pharmacore led to new drugs such as the p56lck inhibitor ${ }^{5}$ and p38 $\alpha$ MAP kinase inhibitor. ${ }^{6}$ Also, selective targeting T-and B-cell lymphomas by 2-aminobenzothiazole moiety has been reported. ${ }^{7}$

Therefore, an efficient and practical method for the synthesis of a diverse collection of $\mathrm{N}$-aryl-2aminobenzazoles would be of great value for drug discovery. The most common method for the synthesis of 2 -aminobenzoxazoles is through the cyclodesulfurization of $N$-substituted-2-hydroxy phenyl-thioureas.

Thiourea intermediates are easily derived from the condensation of an appropriately substituted

\footnotetext{
*For correspondence
}

$o$-aminophenol and any of a number of synthetically or commercially available arylisothiocyanates. ${ }^{8}$ Several cyclodesulfurization methods have appeared in the literature. Procedures, which incorporate reagents such as $\mathrm{HgO},{ }^{9} \mathrm{NiO}_{2},{ }^{10}$ and $\mathrm{LiOH} / \mathrm{H}_{2} \mathrm{O}_{2}{ }^{11}$ and $1,1^{\prime}$-(ethane-1,2diyl)dipyridinium bistribromide (EDPBT) ${ }^{12}$ have been reported.

Further, transition metal-catalyzed (particularly palladium, copper and iron) intramolecular cyclisation of 2-bromobenzothioureas is the most common method for the synthesis of $\mathrm{N}$-aryl-2-aminobenzo- thiazoles. ${ }^{13}$ Synthesis of 2-aminobenzothiazoles via intramolecular C-S bond formation/C-H bond functionalization utilizing a co-catalytic system has been reported. ${ }^{14}$

However, the utility and applicability of these reactions are limited due to toxic metals, expensive catalyst system and pre-functionalization of the starting material. Apart from these, the synthesis of isothiocyanates requires the use of toxic thiophosgene.

Moreover, a disadvantage with isothiocyanates is that they are unstable if stored for long periods. These drawbacks make the presently available methodologies difficult for large in scale industrial application. The present studies to the development of a new route for the synthesis of organic compounds and our interest remains in dithiocarbamate mediated reactions. ${ }^{15}$

\section{Experimental}

\subsection{General experimental}

A mixture of $O$-aminophenol/o-aminothiophenol (1.0 eq), dithiocarbamate (1.2 eq), and $\mathrm{K}_{2} \mathrm{CO}_{3}$ (3.0 eq) 
in dry DMF $(10-15 \mathrm{~mL})$ in $50 \mathrm{~mL}$ rb flask connected to a reflux condensor was refluxed for $2 \mathrm{~h}$. Progress of the reaction was monitored by TLC. The reaction mixture was filtered through celite. The filtrate was concentrated in vacuo and the resulting mixture chromatographed on silica gel (DCM:MeOH) to yield $N$-aryl-2-aminobenzazoles.

\subsection{Experimental data}

2.2a Benzooxazol-2-yl-phenyl-amine (table 1, Entry 1): Light brown solid; M.p.: $142-143^{\circ} \mathrm{C} ;{ }^{1} \mathrm{H}$ NMR (400 MHz, DMSO- $\left.d_{6}\right): \delta 10.60$ (br s, $\left.1 \mathrm{H}, \mathrm{NH}\right), 7.75(\mathrm{~d}, J=$ $7.76 \mathrm{MHz}, 2 \mathrm{H}), 7.44$ (t, $J=7.96 \mathrm{~Hz}, 2 \mathrm{H}), 7.38-7.32$ $(\mathrm{m}, 2 \mathrm{H}), 7.21(\mathrm{t}, J=7.47 \mathrm{~Hz}, 1 \mathrm{H}), 7.12(\mathrm{t}, J=7.62$ $\mathrm{Hz}, 1 \mathrm{H}), 7.03$ (t, $J=7.32 \mathrm{~Hz}, 1 \mathrm{H}$ ); Mass (ESI): 211.0 $[\mathrm{M}+\mathrm{H}]^{+}$.

2.2b Benzothiazol-2-yl-phenyl-amine (table 1, Entry 2): Colourless solid; M.p.: $166-167^{\circ} \mathrm{C}$; IR $\left(\mathrm{KBr}, \mathrm{cm}^{-1}\right)$ : 3444, 3054, 1666, 1573, 1467, 1249; ${ }^{1} \mathrm{H}$ NMR (400 $\left.\mathrm{MHz}, \mathrm{DMSO}-d_{6}\right): \delta 10.46$ (br s, $\left.1 \mathrm{H}, \mathrm{NH}\right), 7.81-7.78$ (m, 3H), 7.61-7.59 (m, 1H), 7.38-7.30 (m, 3H), 7.17$7.714(\mathrm{~m}, 1 \mathrm{H}), 7.04-7.00(\mathrm{~m}, 1 \mathrm{H}) ;{ }^{13} \mathrm{C}$ NMR $(50 \mathrm{MHz}$, DMSO- $\left.d_{6}\right): \delta 161.92,152.46,140.98,130.33,129.34$, 126.21, 122.62, 122.40, 121.39, 119.56, 118.11; Mass (ESI): $227.2[\mathrm{M}+\mathrm{H}]^{+}$.

2.2c Benzooxazol-2-yl-(3-chloro-phenyl)-amine (table 1, Entry 3): Yellow solid; M.p.: $212-213^{\circ} \mathrm{C}$; IR ( $\mathrm{KBr}$, $\mathrm{cm}^{-1}$ ): 3444, 3032, 1663, 1576, 1492, 1231, 1092; ${ }^{1} \mathrm{H}$ NMR (400 MHz, DMSO- $\left.d_{6}\right): \delta 10.50$ (br s, $\left.1 \mathrm{H}, \mathrm{NH}\right)$, 8.15-8.13 (m, 1H), 7.65-7.61 (m, 1H), 7.45-7.05 (m, $6 \mathrm{H})$; Mass (ESI): $244.9[\mathrm{M}+\mathrm{H}]^{+}$.

2.2d Benzothiazol-2-yl-(3-chloro-phenyl)-amine (table 1, Entry 4): solid; M.p.: $237-238^{\circ} \mathrm{C}$; IR $\left(\mathrm{KBr}, \mathrm{cm}^{-1}\right)$ : 3444, 3117, 1620, 1493, 1275, 1098, 730; ${ }^{1} \mathrm{H}$ NMR $\left(400 \mathrm{MHz}, \mathrm{DMSO}-d_{6}\right): \delta 10.61(\mathrm{br} \mathrm{s}, 1 \mathrm{H}, \mathrm{NH}), 7.81(\mathrm{~d}$, $J=8.64 \mathrm{~Hz}, 3 \mathrm{H}), 7.60(\mathrm{~d}, J=7.92 \mathrm{~Hz}, 1 \mathrm{H}), 7.40(\mathrm{~d}$, $J=8.76 \mathrm{~Hz}, 2 \mathrm{H}), 7.32(\mathrm{t}, J=7.28 \mathrm{~Hz}, 1 \mathrm{H}), 7.16(\mathrm{t}$, $J=7.44 \mathrm{~Hz}, 1 \mathrm{H}$ ); Mass (ESI): $261.0[\mathrm{M}+\mathrm{H}]^{+}$.

2.2e Benzooxazol-2-yl-(4-fluoro-phenyl)-amine (table 1, Entry 5): solid; M.p.: $112-113^{\circ} \mathrm{C} ;{ }^{1} \mathrm{H}$ NMR $(400 \mathrm{MHz}$, DMSO- $\left.d_{6}\right): \delta 10.62$ (br s, $\left.1 \mathrm{H}, \mathrm{NH}\right), 7.77-7.73(\mathrm{~m}, 2 \mathrm{H})$, 7.47-7.41 (m, 2H), 7.22-7.09 (m, 4H); ${ }^{13} \mathrm{C}$ NMR (100 $\left.\mathrm{MHz}, \mathrm{DMSO}-d_{6}\right): \delta 158.63,158.00,156.26,147.00$, $135.15,123.98,121.64,119.18,119.10,116.54$, 115.64, 115.41, 108.92; Mass (ESI): $228.8[\mathrm{M}+\mathrm{H}]^{+}$.

2.2f Benzothiazol-2-yl-(4-fluoro-phenyl)-amine (table 1, Entry 6): solid; M.p.: $212-213^{\circ} \mathrm{C}$; IR $\left(\mathrm{KBr}, \mathrm{cm}^{-1}\right)$ :
3443, 3077, 1626, 1574, 1210; ${ }^{1} \mathrm{H}$ NMR (400 MHz, DMSO- $\left.d_{6}\right): \delta 10.49$ (br s, $\left.1 \mathrm{H}, \mathrm{NH}\right), 7.80-7.78(\mathrm{~m}, 3 \mathrm{H})$, $7.57(\mathrm{~d}, J=7.88 \mathrm{~Hz}, 1 \mathrm{H}), 7.31(\mathrm{t}, J=7.56 \mathrm{~Hz}, 1 \mathrm{H})$, 7.22-7.12 (m, 3H); Mass (ESI): $245.0[\mathrm{M}+\mathrm{H}]^{+}$.

2.2g Benzooxazol-2-yl-(4-methoxy-phenyl)-amine (table 1, Entry 7): solid; M.p.: $137-139^{\circ} \mathrm{C}$; IR $\left(\mathrm{KBr}, \mathrm{cm}^{-1}\right)$ : 3383, 3043, 2836, 1674, 1579, 1510, 1232, 1031; ${ }^{1} \mathrm{H}$ NMR (400 MHz, DMSO- $\left.d_{6}\right): \delta 10.60$ (br s, $1 \mathrm{H}$, $\mathrm{NH}), 7.50(\mathrm{~d}, J=8.60 \mathrm{~Hz}, 1 \mathrm{H}), 7.30-7.10(\mathrm{~m}$, $4 \mathrm{H}), 6.91-6.78(\mathrm{~m}, 3 \mathrm{H}), 3.85$ (s, 3H); Mass (ESI): $241.0[\mathrm{M}+\mathrm{H}]^{+}$.

2.2h Benzothiazol-2-yl-(4-methoxy-phenyl)-amine (table 1, Entry 8): solid; M.p.: $166-167^{\circ} \mathrm{C}$; IR $\left(\mathrm{KBr}, \mathrm{cm}^{-1}\right)$ : 3451, 3184, 2902, 1619, 1567, 1510, 1455, 1251, 1030; ${ }^{1} \mathrm{H}$ NMR (400 MHz, DMSO- $d_{6}$ ): $\delta 10.25$ (br $\mathrm{s}, 1 \mathrm{H}, \mathrm{NH}), 7.75(\mathrm{~d}, J=7.64 \mathrm{~Hz}, 1 \mathrm{H}), 7.66$ $(\mathrm{d}, J=8.6 \mathrm{~Hz}, 2 \mathrm{H}), 7.53(\mathrm{~d}, J=7.84 \mathrm{~Hz}, 1 \mathrm{H}), 7.28$ $(\mathrm{t}, J=7.4 \mathrm{~Hz}, 1 \mathrm{H}), 7.10(\mathrm{t}, J=7.4 \mathrm{~Hz}, 1 \mathrm{H}), 6.94$ (d, $J=8.68 \mathrm{~Hz}, 2 \mathrm{H}), 3.73$ (s, 3H); Mass (ESI): 255.1 $[\mathrm{M}+\mathrm{H}]^{+}$.

2.2i Benzooxazol-2-yl-(2,3-dimethyl-phenyl)-amine (table 1, Entry 9): solid; M.p.: $140-141^{\circ} \mathrm{C}$; IR $\left(\mathrm{KBr}, \mathrm{cm}^{-1}\right)$ : 3445, 3146, 2852, 1664, 1580, 1473, 1264; ${ }^{1} \mathrm{H}$ NMR $\left(400 \mathrm{MHz}, \mathrm{DMSO}-d_{6}\right): \delta 10.22(\mathrm{~s}, 1 \mathrm{H}), 7.78-7.75(\mathrm{~m}$, 1H), 7.40-7.38 (m, 1H), 7.25-7.17 (m, 4H), 7.09-7.05 (m, 1H), $2.36(\mathrm{~s}, 3 \mathrm{H}), 2.28(\mathrm{~s}, 3 \mathrm{H}) ;{ }^{13} \mathrm{C}$ NMR $(50 \mathrm{MHz}$, DMSO- $\left.d_{6}\right): \delta 160.53,148.03,143.17,137.53,137.03$, $130.39,126.74,125.93,124.13,122.16,121.20$, 116.34, 109.05, 20.57, 14.19;

$2.2 \mathrm{j}$ Benzothiazol-2-yl-(2,3-dimethyl-phenyl)-amine (table 1, Entry 10): solid; M.p.: $196-197^{\circ} \mathrm{C}$; IR $\left(\mathrm{KBr}, \mathrm{cm}^{-1}\right)$ : 3440, 3134, 2849, 1613, 1569, 1450; ${ }^{1} \mathrm{H}$ NMR (400 MHz, DMSO- $\left.d_{6}\right): \delta 9.67$ (br s, $\left.1 \mathrm{H}, \mathrm{NH}\right), 7.70(\mathrm{~d}, J=$ $7.68 \mathrm{~Hz}, 1 \mathrm{H}), 7.44$ (dd, $J=7.68,7.89 \mathrm{~Hz}, 2 \mathrm{H}), 7.24$ $(\mathrm{t}, J=7.52 \mathrm{~Hz}, 1 \mathrm{H}), 7.14-7.03(\mathrm{~m}, 3 \mathrm{H}), 2.27$ (s, 3H), $2.15(\mathrm{~s}, 3 \mathrm{H})$; Mass (ESI): $255.0[\mathrm{M}+\mathrm{H}]^{+}$.

2.2k Benzooxazol-2-yl-(4-nitro-phenyl)-amine (table 1, Entry 11): solid; M.p.: $189-190{ }^{\circ} \mathrm{C} ;{ }^{1} \mathrm{H}$ NMR (400 MHz, DMSO- $d_{6}$ ): $\delta 10.93$ (br s, 1H, NH), 8.77 (s, 1H), $8.15(\mathrm{~d}, J=8.90 \mathrm{~Hz}, 1 \mathrm{H}), 7.75(\mathrm{~d}, J=8.95 \mathrm{~Hz}, 1 \mathrm{H})$, 7.49-7.40 (m, 2H), $7.28(\mathrm{~d}, J=3.10 \mathrm{~Hz}, 1 \mathrm{H}), 7.15(\mathrm{t}$, $J=4.70 \mathrm{~Hz}, 1 \mathrm{H}), 7.04(\mathrm{t}, J=5.51 \mathrm{~Hz}, 1 \mathrm{H})$; Mass (ESI): $256.0[\mathrm{M}+\mathrm{H}]^{+}$.

2.21 Benzooxazol-2-yl-(3,4-dichloro-phenyl)-amine (table 1, Entry 12): solid; M.p.: $217-218^{\circ} \mathrm{C}$; IR $\left(\mathrm{KBr}, \mathrm{cm}^{-1}\right)$ : $3384,3036,1688,1594,1460,1362,1250,747 ;{ }^{1} \mathrm{H}$ NMR (400 MHz, DMSO- $d_{6}$ ): $\delta 10.30$ (br s, $1 \mathrm{H}$ ), 8.05$8.03(\mathrm{~m}, 1 \mathrm{H}), 7.69-7.63(\mathrm{~m}, 1 \mathrm{H}), 7.44-7.42(\mathrm{~m}, 2 \mathrm{H})$, 
Table 1. Synthesis of $N$-phenyl-2-aminobenzazoles in absence of catalyst.

\begin{tabular}{|c|c|c|c|c|}
\hline Entry & $o$-aminophenol/o-aminothiophenol & Dithiocarbamate & Product & Yield (\%) \\
\hline 1 & $\mathrm{OH}$ & Mes' & & 88 \\
\hline 2 & SH & Mes & & 92 \\
\hline 3 & $\mathrm{OH}$ & & & 84 \\
\hline 4 & SH & & & 86 \\
\hline 5 & OH & Mes' & & 85 \\
\hline 6 & $\mathrm{SH}$ & Mes & & 90 \\
\hline 7 & $\mathrm{OH}$ & Mes & & 82 \\
\hline 8 & SH & & & 85 \\
\hline 9 & $\mathrm{OH}$ & & & 94 \\
\hline 10 & SH & Mes & & 90 \\
\hline 11 & $\mathrm{OH}$ & Mes' & & 84 \\
\hline 12 & $\mathrm{OH}$ & & & 88 \\
\hline 13 & & Mes' & & 86 \\
\hline 14 & SH & & & 92 \\
\hline
\end{tabular}


7.37-7.25 (m, 2H), 7.22-7.18 (m, 1H), 7.06-7.03 (m, 1H); Mass (ESI): $278.9[\mathrm{M}+\mathrm{H}]^{+}$.

2.2m Benzo[1,3]dioxol-5-yl-benzooxazol-2-yl-amine (table 1, Entry 13): solid; M.p.: $207-209^{\circ} \mathrm{C} ;{ }^{1} \mathrm{H}$ NMR (400 MHz, DMSO- $d_{6}$ ): $\delta 10.47$ (br s, $1 \mathrm{H}, \mathrm{NH}$ ), 7.47-7.41 (m, 3H), 7.20-7.08 (m, 3H), 6.93-6.91 (d, $J=8.40$ $\mathrm{Hz}, 1 \mathrm{H}), 6.00$ (s, 2H); Mass (ESI): $255.2[\mathrm{M}+\mathrm{H}]^{+}$.

2.2n Benzothiazol-2-yl-pyridin-2-yl-amine (table 1, Entry 14): Brown solid; M.p.: $233-234^{\circ} \mathrm{C}$; ${ }^{1} \mathrm{H}$ NMR (400 MHz; DMSO-d $\left.{ }_{6}\right): \delta=\delta 11.54(\mathrm{~s}, 1 \mathrm{H}) 8.33(\mathrm{~d}, 1 \mathrm{H}$, $J=7.4 \mathrm{~Hz}) 7.88(\mathrm{~d}, 1 \mathrm{H}, J=7.7 \mathrm{~Hz}) 7.76(\mathrm{t}$, $1 \mathrm{H}, J=8.1, \mathrm{~Hz}), 7.61(\mathrm{~d}, 1 \mathrm{H}, J=7.9 \mathrm{~Hz})$, $7.36(\mathrm{t}, 1 \mathrm{H}, J=7.5 \mathrm{~Hz}), 7.20-7.15(2 \mathrm{H}, \mathrm{m}), 7.00$ $(\mathrm{t}, 1 \mathrm{H}, J=5.7 \mathrm{~Hz}) ;{ }^{13} \mathrm{C} \mathrm{NMR}\left(50 \mathrm{MHz} ; \mathrm{CDCl}_{3}\right)$ : $\delta=159.73,152.00,149.79,146.92,138.63,131.99$, 126.06, 122.33, 121.51, 119.45, 117.21, 111.58, Mass (ESI): $228[\mathrm{M}+\mathrm{H}]^{+}$.

2.2o N-(5-Chloro-benzooxazol-2-yl)-N'-\{6-methoxy-7-[3(4-methyl-piperazin-1-yl)-propoxy]-quinazolin-4-yl\}-benzene-1,4-diamine (5a): Colourless solid; M.p.: 174$176^{\circ} \mathrm{C}$; IR $\left(\mathrm{KBr}, \mathrm{cm}^{-1}\right)$ : 3325, 2939, 2808, 1240,
1568, 1145, 798; ${ }^{1} \mathrm{H}$ NMR (400 MHz, DMSO- $\left.d_{6}\right): \delta$ $10.77(\mathrm{~s}, 1 \mathrm{H}), 9.49(\mathrm{~s}, 1 \mathrm{H}), 8.41(\mathrm{~s}, 1 \mathrm{H}), 7.84(\mathrm{~s}, 1 \mathrm{H})$, $7.74(\mathrm{~s}, 4 \mathrm{H}), 7.51(\mathrm{~d}, J=8.4 \mathrm{~Hz}, 2 \mathrm{H}), 7.16-7.13$ $(\mathrm{m}, 2 \mathrm{H}), 4.16(\mathrm{t}, J=6.0 \mathrm{~Hz}, 2 \mathrm{H}), 3.96(\mathrm{~s}, 3 \mathrm{H})$, 2.46-2.33 (m, 10H), 2.15 (s, 3H), 1.96-1.92 (m, 3H); ${ }^{13} \mathrm{C}$ NMR (50 MHz, DMSO- $d_{6}$ ): $\delta 159.24,156.42$, $153.44,152.92,148.84,146.82,145.92,144.17$, $134.24,134.01,128.11,123.46,121.08,117.98$, 116.13, 109.93, 108.69, 107.72, 102.02, 66.70, 56.21, 54.71, 54.28, 52.65, 45.66, 40.12, 26.01; Mass (ESI): $574.3[\mathrm{M}+\mathrm{H}]^{+}$.

2.2p N-[6-Methoxy-7-(3-morpholin-4-yl-propoxy)-quinazolin-4-yl]- $N^{\prime}$-oxazolo[4,5-b]pyridin-2-yl-benzene-1,4diamine $(5 b)$ : Yellow solid; IR $\left(\mathrm{KBr}, \mathrm{cm}^{-1}\right)$ : 3032 , 2686, 1643, 1564, 1278; ${ }^{1} \mathrm{H}$ NMR (400 MHz, DMSO$\left.d_{6}\right): \delta 11.12(\mathrm{~s}, 1 \mathrm{H}), 9.75(\mathrm{~s}, 1 \mathrm{H}), 8.74(\mathrm{~s}, 1 \mathrm{H})$, 8.25-8.24 (m, 1H), $8.09(\mathrm{~s}, 1 \mathrm{H}), 7.89-7.70(\mathrm{~m}, 5 \mathrm{H})$, 7.25-7.13 (m, 2H), 4.26-4.23 (m, 2H), 3.98-3.71 (m, $13 \mathrm{H}), 2.45-2.17(\mathrm{~m}, 2 \mathrm{H})$; Mass (ESI): $528.3[\mathrm{M}+\mathrm{H}]^{+}$.

2.2q $\quad N^{2}$-(5-Chloro-benzooxazol-2-yl)- $N^{5}$-[6-methoxy-7(3-morpholin-4-yl-propoxy)-quinazolin-4-yl]-pyridine2,5-diamine (5c): Light yellow solid; IR $\left(\mathrm{KBr}, \mathrm{cm}^{-1}\right)$ :

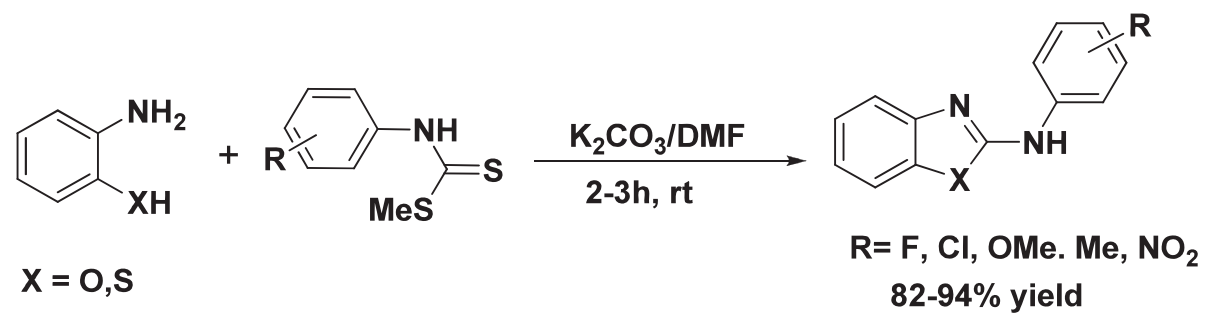

Scheme 1. Preparation of a series of $N$-aryl-2-amino-benzazoles.

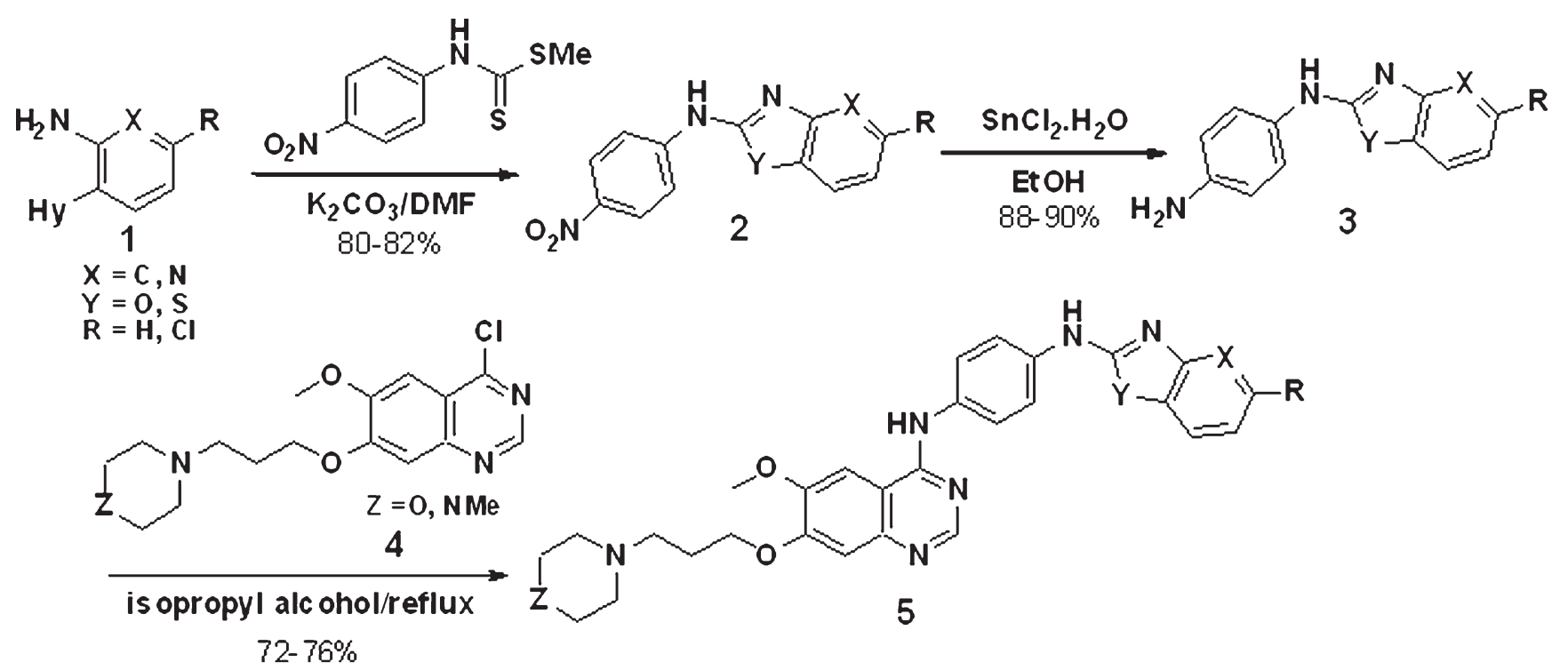

Scheme 2. Synthesis of 4-aminoquinazoline derivatives from $N$-phenyl-2-aminobenzazoles. 
3203, 2953, 2812, 1647, 1533, 1247, 794; ${ }^{1} \mathrm{H}$ NMR $\left(400 \mathrm{MHz}, \mathrm{DMSO}-d_{6}\right): \delta 11.45(\mathrm{~s}, 1 \mathrm{H}), 9.62(\mathrm{~s}, 1 \mathrm{H})$, $8.73(\mathrm{~s}, 1 \mathrm{H}), 8.44(\mathrm{~m}, 1 \mathrm{H}), 8.26(\mathrm{~m}, 2 \mathrm{H}), 7.84(\mathrm{~m}, 1 \mathrm{H})$, 7.57-7.55 (m, 2H), 7.21-7.18 (m, 2H), 4.21-4.18 (m, $2 \mathrm{H}), 3.97(\mathrm{~s}, 3 \mathrm{H}), 3.59(\mathrm{~m}, 4 \mathrm{H}), 2.67-2.32(\mathrm{~m}, 4 \mathrm{H})$, 1.97 (s, 2H); Mass (ESI): $562.3[\mathrm{M}+\mathrm{H}]^{+}$.

\section{2r $\quad N^{2}$-Benzooxazol-2-yl- $N^{5}$-[6-methoxy-7-(3-morpho-}

lin-4-yl-propoxy)-quinazolin-4-yl]-pyridine-2,5-diamine $(5 d)$ : solid; IR $\left(\mathrm{KBr}, \mathrm{cm}^{-1}\right): 3410,2966,2819$, 1625, 1244; ${ }^{1} \mathrm{H}$ NMR (400 MHz, DMSO- $\left.d_{6}\right): \delta 11.27$ $(\mathrm{s}, 1 \mathrm{H}), 9.67(\mathrm{~s}, 1 \mathrm{H}), 8.72-8.71(\mathrm{~s}, 1 \mathrm{H}), 8.46(\mathrm{~m}, 1 \mathrm{H})$, $8.32-8.30(\mathrm{~m}, 1 \mathrm{H}), 8.20-8.22(\mathrm{~m}, 1 \mathrm{H}), 7.88(\mathrm{~s}, 1 \mathrm{H})$, 7.54-7.49 (m, 2H), 7.28-7.15 (m, 3H), 4.26-4.23 $(\mathrm{m}, 2 \mathrm{H}), 3.98-3.71(\mathrm{~m}, 7 \mathrm{H}), 3.32(\mathrm{~s}, 3 \mathrm{H}), 2.49-2.45$ $(\mathrm{m}, 2 \mathrm{H})$; Mass (ESI): $528.2[\mathrm{M}+\mathrm{H}]^{+}$. 2.2s N-(5-Chloro-benzooxazol-2-yl)-N'-[6-methoxy7-(3-morpholin-4-yl-propoxy)-quinazolin-4-yl]-benzene1,4-diamine (5e): yellow solid; IR $\left(\mathrm{KBr}, \mathrm{cm}^{-1}\right)$ : 3423, 2943, 1674, 1514, 1234, 804; ${ }^{1} \mathrm{H}$ NMR (400 MHz, DMSO- $\left.d_{6}\right): \delta 10.88(\mathrm{~s}, 1 \mathrm{H}), 10.23(\mathrm{~s}, 1 \mathrm{H}), 8.59(\mathrm{~s}, 1 \mathrm{H})$, $8.05(\mathrm{~s}, 1 \mathrm{H}), 7.81-7.71(\mathrm{~m}, 4 \mathrm{H}), 7.54-7.51(\mathrm{~m}, 2 \mathrm{H})$, 7.27 (s, 1H), 7.18-7.15 (m, 1H), 4.29-4.26 (m, 2H), 3.99 (s, 7H), 3.32 (s, 3H), 2.28 (m, 2H); Mass (ESI): $561.2[\mathrm{M}+\mathrm{H}]^{+}$.

\section{Results and Discussions}

We are particularly interested in the synthesis of $N$-aryl-2-aminobenzoxazoles and $N$-aryl-2-aminobenzothiazoles via a method suitable for large scale

Table 2. Assay of Aminoquinazolines with Aurora-A kinase data.

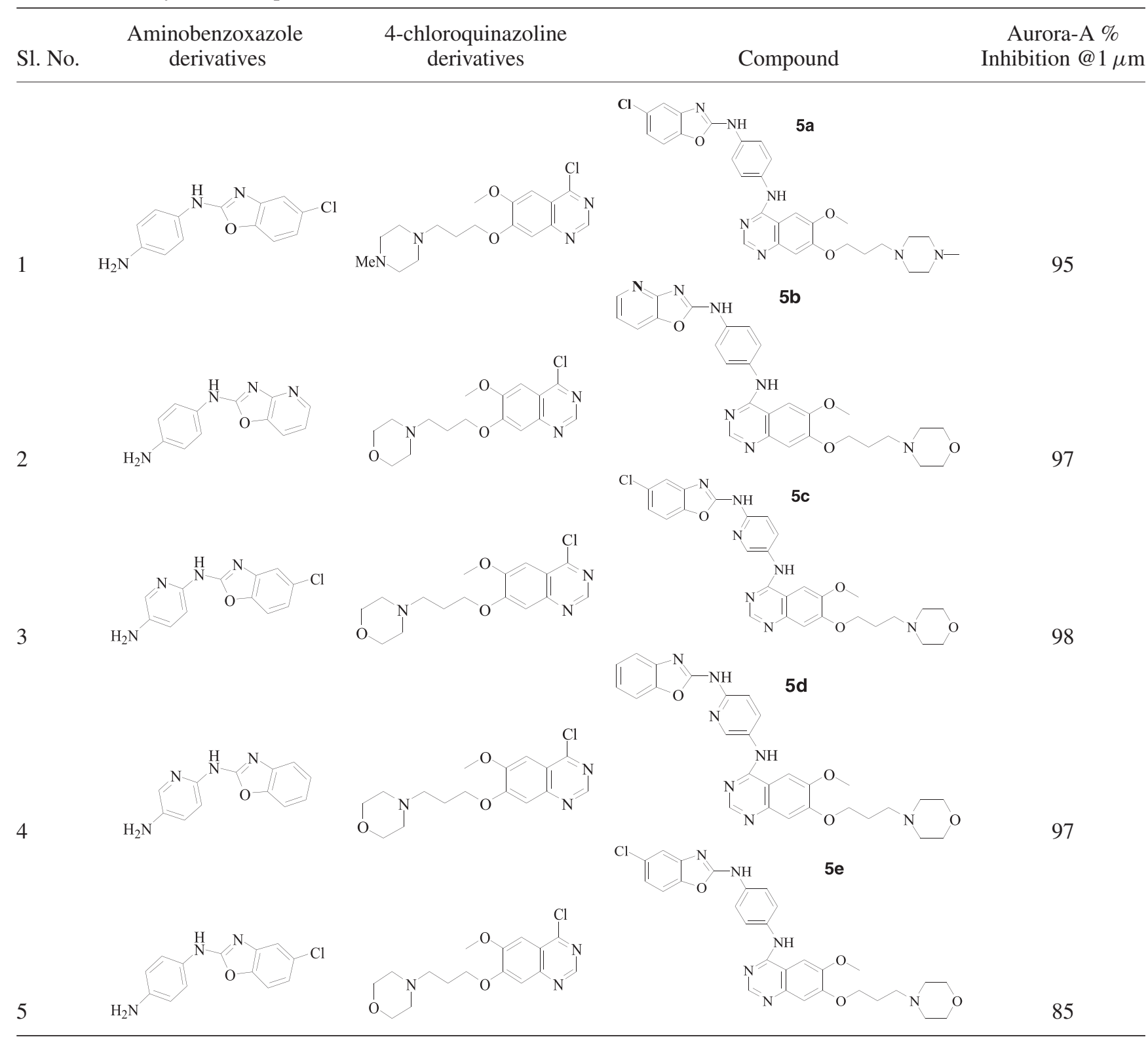


preparations that will not use toxic starting materials or reagents. Herein, we report an efficient onepot synthesis of $\mathrm{N}$-aryl-2-aminobenzazoles by using $o$-aminophenol or $o$-aminothiophenol and substituted dithiocarbamate at room temperature (scheme 1). Unlike isothiocyanates, dithiocarbamates are not toxic, highly stable and easy to handle. ${ }^{16}$ They are easy to synthesize in large quantities using readily available substituted anilines.

The initial experiment involved a reaction between $o$-aminophenol and methyl phenyldithiocarbamate at room temperature yielded without using any catalyst the desired compound of $\mathrm{N}$-phenyl-2aminobenzoxazole. With these encouraging results we started our investigation with different dithocarbamates and $o$-aminophenol/o-aminothiophenol and the results are listed in table 1 . Several dithiocarbamates underwent the above conversion to form a series of $N$-aryl2-amino- benzazoles. The reaction conditions are mild and the experimental procedure is simple. The products were formed in high yields (82-94\%) regardless of the electronic properties of the dithiocarbamates.

Having demonstrated the generality of this reaction in between $o$-aminophenol/o-aminothiophenol and different dithiocarbamates, we further explored the possibility of synthesizing novel 4-aminoquinazoline class of compounds as Aurora kinase-A inhibitor. For this purpose 4-chloroquinazoline derivatives $(\mathbf{4})^{17}$ were synthesized and treated with various $N$-aryl-2aminobenzoxazoles/ $N$-aryl-2-aminobenzothiazoles (3) to yield 4-aminoquinazoline derivatives (5) (scheme 2).

The novel 4-aminoquinazoline derivatives synthesized in this way were tested in Aurora kinase- $\mathrm{A}^{18}$ biological assay and the results are listed in table 2 .

\section{Conclusions}

We have developed a general and efficient one-pot synthesis of $N$-phenyl-2-aminobenzazoles. Mild reaction condition, large scale synthesis, easy and quick isolation of the products and excellent yields are the main advantages of this procedure which makes this method an attractive and useful addition to the present methodologies. Application of this methodology has been demonstrated in synthesizing potent Aurora-A kinase inhibitors.

\section{Supplementary Information}

NMR, IR and mass spectra of the synthesized compounds are reported in Supplementary Information available at www.ias.ac.in/chemsci.

\section{Acknowledgments}

The authors are very thankful to analytical department of Dr. Reddy's Laboratories Ltd. for the spectral and analytical data.

\section{References}

1. (a) Harris N V and Fenton G 2002 World Pat. 2002098426; (b) Darque A, Dumetre A, Hutter S, Casano G, Robin M, Pannecouque C and Azas N 2009 Bioorg. Med. Chem. Lett. 195962

2. Martin R E, Mohr P, Maerki H P, Guba W, Kuratli C, Gavelle O, Binggeli A, Bendels S, Alvarez-Sanchez R, Alker A, Polonchuk L and Christ A D 2009 Bioorg. Med. Chem. Lett. 196106

3. (a) Muro F, Limura S, Yoneda Y, Chiba J, Watanabe T, Masaki S, Takayama G, Yokoyama M, Takashi T, Nakayama A and Machinaga N 2009 Bioorg. Med. Chem. 17 1232; (b) Song H, Oh S R, Lee H K, Han G, Kim J H, Chang H W, Doh K E, Rhee H K and Choo Y P 2010 Bioorg. Med. Chem. 187580

4. Potashman M H, Bready J, Coxon A, DeMelfi Jr. T M, DiPietro L, Doerr N, Elbaum D, Estrada J, Gallant P, Germain J, Gu Y, Harmange J C, Kaufman S A, Kendell R, Kim J L, Kumar G N, Long A M, Neervannan S, Patel V F, Polverino A, Rose P, Plas S ven der, Douglas W, Zanon R and Zhao H 2007 J. Med. Chem. 504351

5. Das J, Moquin R V, Lin J, Doweyko H M, Defex H F, Fung Q, Pang S, Pitt S, Shen D R, Schieven G L, Barrish J C and Wityak J 2003 Bioorg. Med. Chem. Lett. 13 2587

6. Liu C, Lin J, Pitt S, Zhang R F, Sack J S, Kieter S E, Kish K, Doweyko A M, Zhang H, Marathe P H, Trzaskos J, Mckinnon M, Dodd J H, Barrish J C, Schieven G L and Lefthenis K 2008 Bioorg. Med. Chem. Lett. 18 1874

7. Carpenter $\mathrm{R} \mathrm{D}$, Andrei $\mathrm{M}$, Aina $\mathrm{O} \mathrm{H}$, Lau $\mathrm{H} \mathrm{Y}$, Lightstone F C, Liu R, Lam K S and Kurth M J 2009 J. Med. Chem. 5214

8. (a) Ozaki S 1972 Chem Rev. 72 457; (b) Ogura H, Mineo S and Nakagawa K 1981 Chem. Pharm. Bull. 29 1518; (c) Tian Z, Plata D J, Wittenberger S J and Bhatia A V 2005 Tetrahedron Lett. 468341

9. Qian X H, Li Z B, Song G H and Li Z 2001 J. Chem. Res. Synop. 4138

10. Ogura H, Mineo S and Nakagawa K 1981 Chem. Pharm. Bull. 291518

11. Cee V J and Downing N S 2006 Tetrahedron Lett. 47 3747

12. Yella R and Patel B K 2010 J. Comb. Chem. 12754

13. (a) Qui J W, Zhang X G, Tang R Y, Zhong P and Li J H 2009 Ad. Synth. Catal. 351 2319; (b) Wang J, Peng F, Jiang J L, Lu Z J, Wang L Y, Bai J and Pan Y 2008 Tetrahedron Lett. 49 467; (c) Joyce L L, Evindar G and Batey R A 2004 Chem. Comm. 446; (d) Benedı C, Bravo F, Uriz P, Fernández E, Claver C and Castillón S 2003 Tetrahedron Lett. 446073

14. Joyce L L and Batey R A 2009 Org. Lett. 112792 
15. (a) Das P, Kirankumar C, Nareshkumar K, Innus Md, Iqbal J and Srinivas N 2008 Tetrehedron Lett. 49 922; (b) Nareshkumar K, Sreeramamurthy K, Sandananda P, Mukkanti K and Das P 2010 Tetrahedron Lett. 51 899

16. (a) Azizi N, Ebrahimi F, Aakbari E, Aryansab F and Saidi M R 2007 Synthesis 2797; (b) Azizi N, Aryanasab F, Torkiyan L, Ziyaei A and Saidi M R 2006 J. Org. Chem. 71 3634; (c) Azizi N, Aryanasab F and Saidi M R 2006 Org. Lett. 8 5275; (d) Wang Y Q, Ge Z M,
Hou X L, Cheng T M and Li R T 2004 Synthesis 675; (e) Salvatore R N, Sahab S and Jung K 2001 Tetrahedron Lett. 422055

17. (a) Ple P A, Green T P, Hennequin L F, Curwen J, Fennel M, Allen J, van der Brempt C L and Costello G $2004 \mathrm{~J}$. Med. Chem. 47 871; (b) Sreeramamurthy K, Ashok E, Mahendar V, Santoshkumar G and Das P 2010 Synlett. 721

18. Pollard J R and Mortimore M 2009 J. Med. Chem. 52 2629 